\title{
ON A PROBLEM OF J. L. TAYLOR
}

\section{KEIJI IZUCHI}

ABSTRACT. Let $S$ be the structure semigroup of a measure algebra $M(G)$ and $K$ be the union of all maximal groups of $S$. Taylor proposed the following problem: Are there L. C. A. groups $G$ with nontrivial measures concentrated on $K$ ? The purpose of this paper is to give a positive solution to this problem.

Let $G$ be a locally compact abelian group with dual group $\hat{G}$. We denote by $M(G)$ the Banach algebra of all bounded regular Borel measures on $G$ under convolution multiplication and total variation norm. In [2], Taylor showed that there is a compact topological semigroup $S$, called the structure semigroup of $M(G)$, and an order preserving isometry-isomorphism $\theta$ of $M(G)$ into $M(S)$ such that:

(1) $\theta(M(G))$ is a weak ${ }^{*}$-dense $L$-subalgebra of $M(S)$;

(2) the maximal ideal space of $M(G)$ is identified with $\hat{S}$, the set of all continuous semicharacters on $S$, and the Gel'fand transform of $\mu \in M(G)$ is given by $\tilde{\mu}(f)=\int_{S} f d \theta \mu$ for $f \in \hat{S}$. $\{\tilde{\mu}(f) ; f \in \hat{S}\}$ is called the spectrum of $\mu$. $\mu$ is called symmetric if $\widetilde{\mu}^{*}(f)=\overline{\widetilde{\mu}(f)}$ for every $f \in \hat{S}$. Let $K$ be the union of all maximal groups $\left\{K_{p}\right\}_{p \in P}$ of $S$. Then $K=\{x \in S ;|f(x)|=1$ or 0 for every $f \in \hat{S}\}$.

Definition. Let $M_{K}(G)$ be the set of all $\mu \in M(G)$ such that $\theta \mu$ is concentrated on $K$ but $\theta|\mu|\left(K_{p}\right)=0$ for every $p \in P$.

In [3], Taylor proposes the following problem concerning $M_{K}(G)$.

Problem. Are there L. C. A. groups $G$ for which $M_{K}(G) \neq 0$ ?

The purpose of this paper is to show the existence of a L.C.A. group $G$ such that $M_{K}(G) \neq 0$. The following is our main

Theorem. Let $\bar{R}$ be the Bohr compactification of the real line $R$. Then there exists nonzero $\mu \in M_{K}(\bar{R})$ so that $\mu$ is a positive symmetric measure and the spectrum of $\mu$ is a countable set.

We put $\Lambda_{n}=\left\{\left(\alpha_{0}, \alpha_{1}, \ldots, \alpha_{n}\right) ; \alpha_{0}=0, \alpha_{i}=0\right.$ or $\left.1(i=1,2, \ldots, n)\right\}$ $(n=0,1,2, \ldots)$ and $\Lambda=\bigcup_{n=0}^{\infty} \Lambda_{n}$. For $\alpha \in \Lambda$, we put $|\alpha|=n$ if $\alpha \in \Lambda_{n}$.

Lemma 1. There exists a countable family $\left\{E_{\alpha}\right\}_{a \in \mathbf{\Lambda}}$ such that $E_{\alpha}$ is a

Received by the editors October 3, 1974.

AMS (MOS) subject classifications (1970). Primary 43A05, 43A10, 43A32.

Key words and phrases. Structure semigroup, measure algebra, Gel'fand transform, spectrum, symmetric. 
subset of $R(\alpha \in \Lambda)$ satisfying the following conditions:

(1) $E_{a} \subset E_{a, 0}$ and $E_{a} \subset E_{a, 1}$ for $\alpha \in \Lambda$;

(2) $E_{a, 0} \backslash E_{\alpha} \neq \varnothing$ and $E_{a, 1} \backslash E_{a} \neq \varnothing$ for $a \in \Lambda$;

(3) for $a, \beta \in \Lambda, E_{\alpha} \cap E_{\beta}=E_{a_{0}, a_{1}, \ldots, a_{j}}$ if $\alpha_{1}=\beta_{1}, \ldots, \alpha_{j}=\beta_{j}$ and $a_{j+1} \neq \beta_{j+1}$

(4) $\bigcup_{a \in \mathbf{\Lambda}} E_{a}$ is an independent set.

Proof. Since $R$ contains an infinite independent set, it is easy to construct such a family.

We denote by $H_{a}$ the subgroup of $R$ generated by $E_{a}(\alpha \in \Lambda)$. The following lemma is clear by Lemma 1.

Lemma 2. The countable family $\left\{H_{a}\right\}_{a \in \mathbf{\Lambda}}$ has the following properties:

(1) $H_{a} \subset H_{a, 0}$ and $H_{a} \subset H_{a, 1}$ for $a \in \Lambda$;

(2) $H_{a, 0} / H_{a}$ and $H_{a, 1} / H_{a}$ are infinite subgroups for $a \in \Lambda$;

(3) for $\alpha, \beta \in \Lambda, H_{\alpha} \cap H_{\beta}=H_{a_{0}, a_{1}, \ldots, a_{j}}$ if $\alpha_{1}=\beta_{1}, \ldots, \alpha_{j}=\beta_{j}$ and $\alpha_{j+1} \neq \beta_{j+1}$.

Let $G_{\alpha}$ be the annihilator in $\bar{R}$ of $H_{a}(\alpha \in \Lambda)$. We put $G_{\alpha}+G_{\beta}=$ $\left\{x+y ; x \in G_{a}, y \in G_{\beta}\right\}$; then $G_{\alpha}+G_{\beta}$ is a compact subgroup. The following lemma is clear by Lemma 2 .

Lemma 3. $\left\{G_{a}\right\}_{a \in \mathbf{\Lambda}}$ is a family of compact subgroups of $\bar{R}$ and has the following properties:

(1) $G_{\alpha} \supset G_{a, 0}$ and $G_{a} \supset G_{a, 1}$ for $\alpha \in \Lambda$;

(2) $G_{\alpha} / G_{\alpha, 0}$ and $G_{\alpha} / G_{a, 1}$ are compact infinite groups for $\alpha \in \Lambda$;

(3) for $\alpha, \beta \in \Lambda, G_{a}+G_{\beta}=G_{a_{0}, a_{1}, \ldots, a_{j}}$ if $\alpha_{1}=\beta_{1}, \ldots, \alpha_{j}=\beta_{j}$ and $a_{j+1} \neq \beta_{j+1}$.

For a compact subgroup $X \subset \bar{R}$, we denote by $m_{X}$ the normalized Haar measure on $X$. We can consider $m_{X} \in M(\bar{R})$. We put $\mu_{n}=(1 / 2)^{n} \Sigma_{a \in \mathbf{\Lambda}_{n}} m_{G_{\alpha}}$ $(n=0,1, \ldots)$. Then $\mu_{n} \in M(\bar{R}), \mu_{n} \geq 0$ and $\left\|\mu_{n}\right\|=1$. For $\mu \in M(\bar{R})$, we denote by $\hat{\mu}$ the Fourier-Stieltjes transform of $\mu$. By the definition of $\left\{\mu_{n}\right\}_{n=0}^{\infty}$ and Lemma 2, we get

Lemma 4. $\left\{\mu_{n}\right\}_{n=0}^{\infty}$ has the following properties:

(1) If $\gamma \in H_{0}$, then $\hat{\mu}_{n}(\gamma)=1$ for $n=0,1,2, \ldots$;

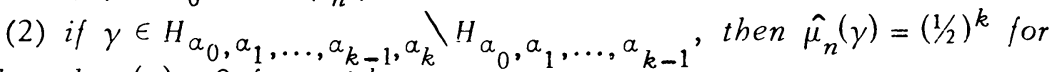
$n \geq k$ and $\mu_{n}(\gamma)=0$ for $n<k$

(3) if $\gamma \in R \backslash H_{a}$ for every $a \in \Lambda$, then $\hat{\mu}_{n}(\gamma)=0, n=0,1,2, \ldots$.

By Lemma $4,\left\{\mu_{n}\right\}_{n=0}^{\infty}$ has only one weak*-cluster point $\mu$ in $M(\bar{R})$ and has the following properties.

Lemma 5. (1) $\mu \in M(\vec{R}), \mu \geq 0$ and $\|\mu\|=1$;

(2) if $\gamma \in H_{0}$, then $\hat{\mu}(\gamma)=1$; 


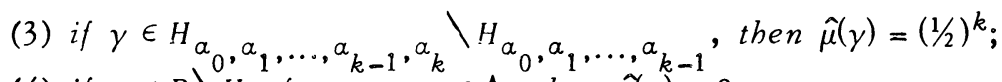

(4) if $\gamma \in R \backslash H_{a}$ for every $\alpha \in \Lambda$, then $\hat{\mu}(\gamma)^{k-1}=0$.

For $a \in \Lambda$, we put $\Lambda_{n}^{a}=\left\{\beta \in \Lambda_{n} ; \alpha_{0}=\beta_{0}, \ldots, \alpha_{|a|}=\beta_{|a|}\right\}$ for $n \geq|a|$ and $\Lambda^{\alpha}=\bigcup_{n \geq|\alpha|} \Lambda_{n^{\circ}}^{\alpha}$. We put $\mu_{n}^{\alpha}=\Sigma_{\beta \in \Lambda_{n}^{\alpha}}(1 / 2)^{n} m_{G_{\beta}}$ for $n \geq|\alpha|$. Then $\mu_{n}^{a} \geq 0$, $\left\|\mu_{n}^{a}\right\|=(1 / 2)^{|a|}$ and $\left\{\mu_{n}^{a}\right\}_{n=|a|}^{\infty}$ has only one weak*-cluster point $\mu^{a}$ in $M(\bar{R})$, and $\left\{\mu^{\alpha}\right\}_{\alpha \in \mathbf{\Lambda}}$ has the following properties.

Lemma 6. (1) $\mu_{n}=\Sigma_{a \in \Lambda_{n}} \mu_{n}^{a}$;

(2) if $\gamma \in H_{\alpha}$, then $\widehat{\mu^{\alpha}}(y)=(1 / 2)|\alpha|$;

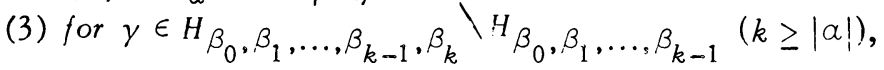

$$
\begin{aligned}
\widehat{\mu^{\alpha}}(\gamma) & =(1 / 2)^{k} \quad \text { if }\left(\beta_{0}, \beta_{1}, \ldots, \beta_{k}\right) \in \Lambda^{\alpha}, \\
& =0 \quad \text { if }\left(\beta_{0}, \beta_{1}, \ldots, \beta_{k}\right) \notin \Lambda^{\alpha},
\end{aligned}
$$

(4) if $\gamma \in R \backslash H_{\alpha}$ for every $\alpha \in \Lambda$, then $\widehat{\mu^{\alpha}}(\gamma)=0$.

For a compact subgroup $X \subset \bar{R}$, there exists the strongest L.C.A. group topology on $\bar{R}$ such that $X$ is an open compact subgroup of $\bar{R}$. We denote by $\bar{R}_{X}$ the resulting L.C. A topological group. We may consider $M\left(\bar{R}_{X}\right) \subset M(\bar{R})$. For $\lambda_{1}, \lambda_{2} \in M(\bar{R})$, we denote by $\lambda_{1} \perp \lambda_{2}$ if $\lambda_{1}$ is mutually singular with $\lambda_{2}$. For $\lambda \in M(\bar{R})$ and a subset $N \subset M(\bar{R})$, we denote by $\lambda \perp N$ if $\lambda \perp \nu$ for every $\nu \in N$.

Lemma 7. (1) $\mu=\Sigma_{\alpha \in \mathbf{\Lambda}_{k_{\beta}}} \mu^{\alpha}$ for every positive integer $k$;

(2) $\mu^{\alpha} \in M\left(\bar{R}_{G_{\alpha}}\right)$, and $\mu^{\beta} \perp M\left(\bar{R}_{G_{\alpha}}\right)$ for $\beta \neq \alpha,|\beta|=|\alpha|$;

(3) for $\alpha \neq \beta$,

$$
\mu^{\alpha} * \mu^{\beta}=(1 / 2)|\alpha|_{(1 / 2)}|\beta|_{m_{a_{0}, a_{1}, \ldots, a_{j}}}
$$

if $\alpha_{1}=\beta_{1}, \ldots, \alpha_{j}=\beta_{j}$ and $\alpha_{j+1} \neq \beta_{j+1}$.

Proof. By (3) of Lemmas 2, 5 and 6, we have $\hat{\mu}=\Sigma_{\alpha \in \mathbf{A}_{k}} \widehat{\mu^{\alpha}}$ for every integer $k$, that is $\mu=\Sigma_{a \in \Lambda_{k}} \mu^{\alpha}$. For $\alpha \neq \beta$ such that $\alpha_{1}=\beta_{1}, \ldots, \alpha_{j}=\beta_{j}$ and $\alpha_{j+1} \neq \beta_{j+1}$, we have

$$
\begin{aligned}
\widehat{\mu^{\alpha} * \mu^{\beta}}(\gamma)=\widehat{\mu^{\alpha}}(\gamma) \widehat{\mu}(\gamma) & =(1 / 2)|\alpha|+|\beta| & & \text { if } \gamma \in H_{a_{0}, \ldots, a_{j},} \\
& =0 & & \text { if } \gamma \notin H_{a_{0}, \ldots, a_{j}},
\end{aligned}
$$

by (3) of Lemmas 2 and 6. This shows that

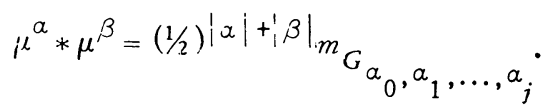

Let $\phi$ be a canonical homomorphism of $\bar{R}$ onto $\bar{R} / G_{a}$, and for $\lambda \in M(\bar{R})$ we put $\dot{\lambda}(E)=\lambda\left(\phi^{-1}(E)\right)$ for every Borel set $E$ of $\bar{R} / G_{\alpha}$. Then $\dot{\lambda} \in M\left(\bar{R} / G_{a}\right)$ and 
$\hat{\lambda}(\gamma \circ \phi)=\hat{\dot{\lambda}}(\gamma)$ for $\gamma \in \widehat{R} / G_{\alpha}=H_{\alpha}$. If $\gamma \in H_{\alpha}\left(\alpha=\left(a_{0}, a_{1}, \ldots, a_{k}\right)\right)$ then $\gamma \epsilon$ $H_{a_{0}, a_{1}, \ldots, a_{j} \backslash H_{a_{0}}, a_{1}, \ldots, a_{j-1}}$ for some $0 \leq j \leq k$ and $\hat{\dot{\mu}}(\gamma)=(1 / 2)^{j}$ by (3) of Lemma 5. Then we have

$$
\dot{\mu}=1 / 2 m_{D_{a_{0}}}+(1 / 2)^{2} m_{D_{a_{0}} \cdot a_{1}}+\cdots+(1 / 2)^{k} m_{D_{a_{0}}, a_{1}, \ldots, a_{k-1}}+(1 / 2)^{k} \delta_{0} .
$$

where $D_{a_{0}, a_{1}, \ldots, a_{j}}$ is the annihilator in $\bar{R} / G_{a}$ of $H_{a_{0}, a_{1}, \ldots, a_{j}} \subset H_{a}$, $m_{D_{a_{0}}, a_{1}, \ldots, a_{j}}$ is the normalized Haar measure on $D_{a_{0}, a_{1}, \ldots, a_{j}}$ and $\delta_{0}$ is the point measure at $0 \in \bar{R} / G_{a}$. Since $H_{\alpha^{\prime}}^{\prime} H_{a_{0}}, a_{1}, \ldots, \alpha_{j}$ is an infinite group, ${ }^{m} D_{\alpha_{0}}, \alpha_{1}, \ldots, a_{j}$ is a continuous measure on $\bar{R} / G_{\alpha}$. Since $\mu_{n}^{a}(n \geq|\alpha|)$ is concentrated on $G_{\alpha}, \mu^{a}$ is concentrated on $G_{a}$ and $\dot{\mu}^{\alpha}=(1 / 2)^{k} \delta_{0}$. Thus $\Sigma_{\beta \neq a, \beta \in \Lambda_{1, a^{\prime}}} \dot{\mu}^{\beta}$ is a continuous measure on $\bar{R} / G_{\alpha}$ and we have $\mu^{a} \in M\left(\bar{R}_{G_{\alpha}}\right)$ and $\mu_{\perp M}{ }^{\beta}\left(\bar{R}_{G_{\alpha}}\right)$ for $\beta \neq \alpha$ and $|\beta|=|\alpha|$.

Remark. By (2) of Lemma 7, $\mu^{\alpha} \perp \mu^{\beta}$ if $\alpha \neq \beta$ and $|\alpha|=|\beta|$.

By Lemma 7, we have

Proposition 1. $O \mu\left(K_{p}\right)=0$ for every maximal group $K_{p}$ of $S$.

Proof. Suppose $\theta \mu\left(K_{p}\right) \neq 0$ for a maximal group $K_{p}$ of $S$. Then there is a positive integer $n$ such that $(1 / 2)^{n}<\theta \mu\left(K_{p}\right)$. By (1) of Lemma 7, there is $\alpha \in \Lambda_{n}$ such that $\theta \mu^{\alpha}\left(K_{p}\right) \neq 0$. By (2) of Lemma 7, we have $\theta \mu^{\beta}\left(K_{p}\right)=0$ for every $\beta \in \Lambda_{n}(\beta \neq \alpha)$. So we have $\theta \mu\left(K_{p}\right)=\theta \mu^{\alpha}\left(K_{p}\right) \leq\left\|\mu^{\alpha}\right\|=(1 / 2)^{n}$, a contradiction.

For $f \in \hat{S}$ and $f^{2}=f$, we put $S_{0}(f)=\{x \in S ; f(x)=0\}, S_{1}(f)=\{x \in S$; $f(x)=1\}$ and $M\left(S_{j}(f)\right)=\left\{\mu \in M(\bar{R}) ; \theta \mu\right.$ is concentrated on $\left.S_{j}(f)\right\}(j=0,1)$. Then $M\left(S_{0}(f)\right)$ is an L-ideal of $M(\bar{R})$ and $M\left(S_{1}(f)\right)$ is an L-subalgebra [2].

Lemma 8. Let $f \in \hat{S}$ such that $f^{2}=f$ and $\tilde{\mu}(f) \neq 0$. Then there exists $\alpha \in \Lambda$ such that:

(1) $\mu^{\alpha} \in M\left(S_{1}(f)\right)$;

(2) $\mu^{\beta} \in M\left(S_{0}(f)\right)$ for $\beta \neq \alpha$ and $|\beta|=|\alpha|$.

Proof. Since $\tilde{\mu}(f) \neq 0$, we can decompose $\mu=\lambda_{1}+\lambda_{2}\left(\lambda_{2} \neq 0\right)$, where $\lambda_{1} \in M\left(S_{0}(f)\right)$ and $\lambda_{2} \in M\left(S_{1}(f)\right)$. Suppose that $\mu_{n} \in M\left(S_{0}(f)\right)$ for every integer $n$. For some integer $n_{0}$ such that $(1 / 2)^{n_{0}}<\left\|\lambda_{2}\right\|$, there exists $\chi, \nu \in \Lambda_{n_{0}}$ such that $\mu^{\boldsymbol{X}} \star \lambda_{2}$ and $\mu^{\nu} \Varangle \lambda_{2}$. Because, $\mu^{\boldsymbol{x}} \perp \mu^{\chi^{\prime}}$ for $\chi, \chi^{\prime} \in \Lambda_{n_{0}}$ and $\chi \neq \chi^{\prime}$, by the remark of Lemma 7 , and $\left\|\mu^{x}\right\|=(1 / 2)^{n_{0}}$ for every $\chi \in \Lambda_{n_{0}}$. By Lemma 7, we have

$$
\mu^{\boldsymbol{x}} * \mu^{\nu}=(1 / 2)^{n} 0(1 / 2)^{n}{ }^{n} m_{\sigma_{0}, \boldsymbol{x}_{1}, \ldots, \boldsymbol{x}_{j}},
$$

where $\chi_{1}=\nu_{1}, \ldots, X_{j}=\nu_{j}$ and $X_{j+1} \neq \nu^{\prime}{ }_{j+1}$, and $\mu^{\boldsymbol{x}} * \mu^{\nu} \epsilon M\left(S_{0}(f)\right)$. Since $\mu^{\boldsymbol{x}} \pm \lambda_{2}$ and $\mu^{\nu} \Varangle \lambda_{2}$, we have $\mu^{\mathbf{x}} * \mu^{\nu} \pm \lambda_{2} * \lambda_{2}$. Since $\lambda_{2} * \lambda_{2} \in M\left(S_{1}(f)\right)$, we have $\mu^{\boldsymbol{x}} * \mu^{\nu} \notin M\left(S_{0}(f)\right)$, a contradiction. Thus the re exists an integer $n$ such that $\mu_{n} \notin M\left(S_{0}(f)\right)$. Let $n_{1}$ be the smallest integer such that $\mu_{n} \notin$ $M\left(s_{0}(f)\right)$. Then there exists $a \in \Lambda_{n_{1}}$ such that $m_{G_{\alpha}} \in M\left(S_{1}(f)\right)$ and $m_{G_{\beta}} \epsilon$ 
$M\left(S_{0}(f)\right)$ for $\beta \in \Lambda_{n_{1}}$ and $\beta \neq \alpha$, by (3) of Lemma 3. Since $M\left(\bar{R}_{G_{a}}\right) \subset$ $M\left(S_{1}(f)\right)$, we have $\mu^{\alpha} \in M\left(S_{1}(f)\right)$ by (2) of Lemma 7. Suppose that $\mu^{\beta} \notin$ $M\left(S_{0}(f)\right)$ for some $\beta \in \Lambda_{n_{1}}$ and $\beta \neq \alpha$. Then we have $\mu^{\beta} * \mu^{\alpha} \notin M\left(S_{0}(f)\right)$. By (3) of Lemma 7, we have

$$
\mu^{\beta} * \mu^{\alpha=(1 / 2)}|\beta|+\left.{ }^{\alpha}\right|_{m_{a_{0}, \alpha_{1}, \ldots, \alpha_{j}},},
$$

where $\alpha_{1}=\beta_{1}, \ldots, \alpha_{j}=\beta_{j}$, and $\alpha_{j+1} \neq \beta_{j+1}$, and $j<|\alpha|=n_{1}$. This shows that $\mu^{a} * \mu^{\beta} \in M\left(S_{0}(f)\right)$, a contradiction. Thus we complete this lemma.

For $f \in \hat{S}, f \geq 0$, there exists $g_{f} \in \hat{S}, g_{f}^{2}=g_{f}$ such that $M\left(S_{1}\left(g_{f}\right)\right)=$ $M(O(f))$, where $O(f)=\{x \in S ; f(x)=1\}$ and $M(O(f))=\{\mu \in M(\bar{R}) ; \theta \mu$ is concentrated on $O(f)\}$ [2].

Proposition 2. $\theta \mu$ is concentrated on $K$.

Proof. Let $f \in \hat{S}$ such that $f \geq 0, f^{2} \neq f$ and $\tilde{\mu}(f) \neq 0$. Let $f=h_{f} \cdot f$ be the polar decomposition of $f$, where $h_{f}^{2}=h_{f} \in \hat{S}$ [2, Lemma 3.3]. Then $\tilde{\mu}\left(h_{f}\right) \neq 0$. By Lemma 8 , there exists $a \in \Lambda$ such that $\mu^{\alpha} \in M\left(S_{1}\left(h_{f}\right)\right)$ and $\mu^{\beta} \in M\left(S_{0}\left(h_{f}\right)\right)$ for $\beta \neq \alpha$ and $|\beta|=|\alpha|$. Since $M\left(\bar{R}_{G_{\alpha}}\right) \subset M\left(S_{1}\left(h_{f}\right)\right)$ and $m_{G_{a}} \in M\left(S_{1}\left(g_{f}\right)\right)$, we have $\left.M\left(\bar{R}_{G_{a}}\right) \subset M\left(g_{f}\right)\right)$ and $\mu^{a} \in M\left(S_{1}\left(g_{f}\right)\right)$ [3]. Thus we complete the proof of this proposition.

Proposition 3. $\mu$ is a symmetric measure and $\mu$ has a countable spectrum.

Proof. Since $\hat{\mu} \geq 0$, we have $\mu^{*}=\mu$. Let $f \in \hat{S}$. By the proof of Proposition 2 , there exists $\alpha \in \Lambda$ such that $\tilde{\mu}(f)=\tilde{\mu}^{\alpha}(f)$. Since $\mu^{\alpha} \in M\left(\bar{R}_{G_{\alpha}}\right)$ and $\tilde{\lambda}(|f|)=\|\lambda\|$ for every positive $\lambda \in M\left(\bar{R}_{G_{a}}\right)$, there exists $\gamma \in \widehat{\bar{R}}_{G_{a}}$ such that $\tilde{\mu}^{\alpha}(f)=\widetilde{\hat{\mu}}^{\alpha}(\gamma)[2]$. Since $\mu^{\alpha} \in M\left(G_{\alpha}\right)$, there exists $\eta \in \hat{G}_{\alpha} \subset R$ such that $\tilde{\mu}(f)$ $=\tilde{\mu}^{\alpha}(f)=\hat{\mu}^{\alpha}(\gamma)=\hat{\mu}^{\alpha}(\eta)$. By Leinma 6 , we have

$$
\left\{\widehat{\mu^{a}} \eta\right) ; \eta \in R_{1}^{l}=\left\{0,(1 / 2)|a|,(1 / 2)^{|\alpha|+1},(1 / 2)^{|\alpha|+2}, \ldots\right\} .
$$

Thus we have $\{\tilde{\mu}(f) ; f \in \hat{S}\}=\left\{0,1,1 / 2,(1 / 2)^{2},(1 / 2)^{3}, \ldots\right\}$. This shows that $\mu$ is symmetric and has a countable spectrum.

By Propositions 1, 2 and 3, we have our Theorem.

Corollary. There is a compact metrizable abelian group $G$ and a nonzero symmetric measure $\mu \in M_{K}(G)$ so that the spectrum of $\mu$ is a countable set.

Proof. We may assume that $E_{\alpha}$ is a countable set $(\alpha \in \Lambda)$ in Lemma 1. Let $H$ be the subgroup generated by $\left\{H_{\alpha}\right\}_{\alpha \in \Lambda}$; then $H$ is a countable subgroup of $R$. Let $H^{\perp}$ be the annihilator of $H$ in $\bar{R}$; then $H^{\perp}$ is a compact subgroup of $\bar{R}$. Since $H=\widehat{\bar{R} / H^{\perp}}, \bar{R} / H^{\perp}$ is a compact metrizable group. Then we can construct $\mu \in M\left(\bar{R} / H^{\perp}\right)$, which has the properties of this corollary, in the same way as in the proof of our Theorem. 


\section{REFERENCES}

1. W. Rudin, Fourier analysis on groups, Interscience $T_{\text {racts }}$ in Pure and $A_{\text {ppl. }}$ Math., no. 12, Interscience, New York, 1962. MR 27 \#2808.

2. J. L. Taylor, The structure of convolution measure algebras, Trans. Amer. Math. Soc. 119 (1965), 150-166. MR 32 \#2932.

3. - L L-subalgebras of $M(G)$, Trans. Amer. Math. Soc. 135 (1969), 105113. MR $38 \# 1472$.

DEPARTMENT OF MATHEMATICS, TOKYO UNIVERSITY OF EDUCATION, TOKYO, J AP AN

Current address: Department of Mathematics, Kanagawa University, Yokohama, Japan 